\title{
Estado de salud de los adultos mayores institucionalizados de la ciudad de Cuenca
}

\section{Health status of institutionalized older adults in the city of Cuenca}

\author{
Nube Johanna Pacurucu Avila*1,2, Edison Gustavo Moyano Brito ${ }^{1,2}$, Katynna Cecilia Parra Quevedo ${ }^{2}$, María \\ Fernanda Peralta Cárdenas ${ }^{1}$, Josué Francisco Aguilar Valenzuela ${ }^{1}$ \\ ${ }^{1}$ Universidad Católica de Cuenca, Ecuador, Ecuador \\ ${ }^{2}$ Universidad del Zulia, Venezuela \\ *npacurucua@ucacue.edu.ec
}

DOI: https://doi.org/10.26871/killkanasalud.v4i4.753

\begin{abstract}
Resumen
Objetivo: Determinar el estado de salud de adultos mayores institucionalizados en el Geriátrico Miguel de León de la Ciudad de Cuenca. Materiales y Métodos: estudio cuantitativo de alcance descriptivo con temporalidad retrospectiva, la muestra estuvo conformada por 44 adultos mayores institucionalizados, la información se obtuvo mediante la revisión de historias clínicas, en donde se registraron datos sociodemográficos, valores sanguíneos como: hemograma, (neutrófilos, glóbulos blancos, glóbulos rojos, linfocitos, monocitos, perfil lipídico(colesterol, triglicéridos, HDL colesterol, LDL colesterol, glicemia) así como el estado nutricional. Las variables de estudio se recopilaron atreves de una ficha de recolección de datos construida por los autores y validada a juicio de expertos. Resultados: en los datos socio demográficos se determinó que, del total de la población, un 47,7\% pertenecen al rango de edad entre 75 a 90 años; en un predominio del sexo femenino con 54,54\%, en lo referente al nivel socioeconómico el 34,09\% son de recursos económicos bajos, y en cuanto al estado civil $56,81 \%$ son viudos, en cuanto al estado nutricional se encontró que el $9,10 \%$, adultos mayores se encuentran en riesgo de desnutrición, el $22,72 \%$ presentó desnutrición y el $68,18 \%$, se encontraron en estado nutricional normal, el perfil lípido se observa que el 38,6\% se encuentran alto en lo que es colesterol. Conclusiones: el estado de salud de los adultos mayores se ve afectado principalmente por enfermedades como la diabetes y colesterol alto, los adultos mayores evaluados se encuentran dentro del rango normal con un $61,4 \%$, y el $38,6 \%$ se encuentran con valores referenciales alterados.
\end{abstract}

Palabras clave: anciano, estado nutricional, diabetes, salud, asilo.

\section{Abstract}

Objective: To determine the health status of institutionalized older adults in the Miguel de Leon nursing home in the City of Cuenca. Materials and Methods: quantitative study of descriptive scope with retrospective temporality, the sample consisted of 44 institutionalized older adults, the information was obtained by reviewing medical records, where sociodemographic data, blood values such as: hemogram, (neutrophils, white blood cells, red blood cells, lymphocytes, monocytes, lipid profile (cholesterol, triglycerides, HDL cholesterol, LDL cholesterol, glycemia) as well as nutritional status. The study variables were collected through a data collection sheet constructed by the authors and validated in the opinion of experts. Results: in the socio-demographic data it was determined that, of the total population, $47.7 \%$ belong to the age range between 75 and 90 years; in a predominance of the female sex with $54.54 \%$, regarding socioeconomic level, $34.09 \%$ are of low economic resources, and regarding marital status, $56.81 \%$ are widowers, regarding status It was found that $9.10 \%$, older adults are at risk of malnutrition, $22.72 \%$ presented malnutrition and $68.18 \%$ were in normal nutritional state, the lipid profile is observed that $38,6 \%$ are high in what is cholesterol. Conclusions: the health status of the elderly is affected mainly by diseases such as diabetes and high cholesterol, of the elderly they are within the normal range with $61.4 \%$, and $38.6 \%$ are high.

Keywords: elderly, nutritional status, diabetes, health, asylum.

\section{Introducción}

El envejecimiento es un proceso interno gradual, complejo e irreversible, que se caracteriza por varios cambios en la forma, bioquímica, psicología y función, que limita la capacidad de los organismos para adaptarse al medio ambiente. El estado nutricional de este grupo vulnerable puede cambiar debido a diferentes condiciones, la más destacada de las cuales es; cambios morfo fisiológicos durante el envejecimiento, cambios en el metabolismo y la nutrición y enfermedades crónico degenerativas. ${ }^{1}$ 
El riesgo nutricional de las personas mayores se presenta después de los 60 años, debido a cambios anatómicos y fisiológicos, como: el descenso gradual de la altura, debido a la desviación del nivel de la columna (lordosis y lordosis), tejido muscular, densidad ósea debilitada y agua corporal. Disminución, pérdida de dientes, atrofia de las papilas gustativas y disminución del metabolismo basal. ${ }^{2}$

En países de Latinoamérica, la prevalencia en la desnutrición de adultos mayores que residen en zonas rurales, varía de 4,6\% y $18 \%$ y en adultos mayores hospitalizados aumenta en un $50 \% .{ }^{3}$ En Argentina la prevalencia de riesgo nutricional alcanzó el 23,3\%, y el $10 \%$ se encontraban malnutridos en 257 sujetos estudiados con edades superiores a 65 años según Lufrano $\mathrm{J}$, et al; en el mismo estudio, de 103 adultos mayores con edad superior a 80 años se encontró que un $30 \%$ se encontraban normo nutridos, $60 \%$ en riesgo nutricional y un $10 \%$ en estado de desnutrición. ${ }^{4}$ En el mismo País, Muñoz-Díaz B, et al, en su estudio, tomo como muestra a 218 adultos mayores de 65 años, donde estableció una prevalencia de desnutrición con un $21.2 \%$ y riesgo de la misma en un $40.1 \%{ }^{5}$.

El deterioro del estado de salud de las personas mayores está relacionado con el desequilibrio de la flora intestinal, ya que tiende a consumir menos alimentos debido a diversos motivos, como la reducción de la contractilidad de los músculos masticatorios y la disminución paulatina de los músculos. Los cambios en los dientes, los umbrales del gusto y el olfato, los cambios en la función motora gástrica reducirán la síntesis y secreción de diferentes sustancias, y la relajación de la pared del estómago provocará una sensación de saciedad más temprana y reducirá el mecanismo de absorción. 6

La anemia se ha convertido en una entidad de suma importancia entre los ancianos por su alta prevalencia y consecuencias clínicas negativas en términos de mortalidad, funcionalidad y calidad de vida. La definición de anemia en los ancianos, que es la más utilizada y aceptada internacionalmente por la comunidad científica, fue establecida por la Organización Mundial de la Salud (OMS). ${ }^{7}$

Dentro de la evaluación general del adulto mayor, es primordial valorar el estado nutricional, debido a que se relaciona directamente en la recuperación y tratamiento de enfermedades crónicas-degenerativas y agudas; la evaluación nutricional se realiza de forma primordial en la atención primaria por parte del personal de salud, teniendo en cuenta la etiología principal de la desnutrición en los adultos mayores valorados ${ }^{8}$.

En los últimos años se han realizado investigaciones en diferentes campos relacionados con las ciencias de la salud, pero dado que es difícil obtener investigaciones sobre el estado nutricional de las personas mayores, existen pocos temas de investigación relacionados con el estado nutricional de las personas mayores. población. Los ancianos lucen saludables, pero el riesgo de desnutrición es alto, por lo que ni la sociedad ni los trabajadores de la salud lo notan. ${ }^{4}$ Por lo anterior, el objetivo principal de este estudio fue determinar los factores de desnutrición en una muestra de adultos mayores del Geriátrico Miguel de León de la Ciudad de Cuenca.

\section{Materiales y Métodos}

El presente estudio se realizó con un enfoque cuantitativo de alcance descriptivo con temporalidad retrospectiva, la población de estudio estuvo conformada por 44 adultos mayores institucionalizados en el Geriátrico Miguel de León de la ciudad de Cuenca. La muestra fue censal debido al tamaño de la población.

La información se obtuvo mediante la revisión de historias clínicas obtenidas durante el periodo enero a diciembre del 2019, previa a la autorización de la directora del Geriátrico Miguel de León en donde se registraron datos sociodemográficos, valores sanguíneos como: hemograma, (neutrófilos, glóbulos blancos, glóbulos rojos, linfocitos, monocitos, perfil lipídico( colesterol, triglicéridos,HDL colesterol, LDL colesterol, glicemia;) así como el estado nutricional que se evaluó según la información recabada de la Historia clínica mediante la aplicación del MNA (Mini Nutritional Assessment). La información se recopilo atreves de una ficha de recolección de datos construida por los autores y validada a juicio de expertos, se realizó un análisis descriptivo mediante tablas y frecuencias de las variables sociodemográficas, el estado nutricional y valores sanguíneos mediante el programa estadístico SPSS versión 15 .

\section{Resultados}

En la tabla 1 se puede observar que la mayor prevalencia de edad en adultos mayores es de 75 a 90 con un porcentaje de $47,7 \%$ siendo las viejas o ancianas las que se encuentran en el Geriátrico Miguel de León; seguidas de los adultos mayores de edad avanzada encontrándose entre 60 - 74 años con un $31,8 \%$ y teniendo con el $20,5 \%$ los adultos de más de 90 años.

Tabla 1: Edad en años de adultos mayores del geriátrico Miguel de León

\begin{tabular}{lcc}
\hline & $\begin{array}{c}\text { Porcentaje } \\
\text { válido }\end{array}$ & $\begin{array}{c}\text { Porcentaje } \\
\text { acumulado }\end{array}$ \\
\hline $\begin{array}{l}\text { 60 a 74 años son consideradas } \\
\text { de edad avanzada }\end{array}$ & 14 & 31,8 \\
75 a 90 viejas o ancianas & 21 & 47,7 \\
90 se les denomina grandes & 9 & 20,5 \\
viejos o grandes longevos & 44 & $100 \%$ \\
TOTAL & & \\
\hline
\end{tabular}

La población adulta mayor que acude al Centro Geriátrico Miguel de León del Cantón Cuenca, según las características sociodemográficas, se determinó que, del total de la población, un $47,7 \%$ pertenecen al rango de edad entre 75 a 90 años; en un predominio del sexo femenino con $54,54 \%$, en lo referente al nivel socioeconómico el $34,09 \%$ son de recursos económicos bajos, y en cuanto al estado civil $56,81 \%$ son viudos (tabla 2 ). 
Tabla 2: Distribución de las variables sociodemográficas de en adultos mayores del geriátrico Miguel de León

\begin{tabular}{llcc}
\hline & Variable & Frecuencia & Porcentaje \\
\hline \multirow{3}{*}{ Edad } & Senectud (60-74 años). & 14 & $31 \%$ \\
& Vejez (75 a 90 años). & 21 & $47,7 \%$ \\
& Longevidad (más de 90 años). & 9 & $20,5 \%$ \\
\hline \multirow{2}{*}{ Economía } & Medio (401 a 800). & 10 & $22,72 \%$ \\
& Bajo (100 a 400). & 15 & $34,09 \%$ \\
\hline \multirow{3}{*}{ Genero } & Sin estudios. & 19 & $43,18 \%$ \\
& Primaria. & 20 & $45,45 \%$ \\
& Secundaria. & 4 & $9,09 \%$ \\
\hline \multirow{3}{*}{ Nivel de instrucción } & Sin estudios. & 19 & $43,18 \%$ \\
& Primaria. & 20 & $45,45 \%$ \\
& Secundaria. & 4 & $9,09 \%$ \\
\hline \multirow{3}{*}{ Estado civil } & Soltero. & 8 & $18,18 \%$ \\
& Casado. & 7 & $15,90 \%$ \\
& Divorciado. & 4 & $9,09 \%$ \\
& Viudo. & 25 & $56,81 \%$ \\
\hline
\end{tabular}

Fuente: Base de datos SPSS

En la tabla 3 se puede observar que en la prevalencia de colesterol de los adultos mayores se encuentran dentro del rango normal con un $61,4 \%$, y el $38,6 \%$ se encuentran alto. Los triglicéridos se encuentran en un porcentaje normal con un $72,7 \%$ y un $27,3 \%$ entre los rangos altos. La prevalencia de HDL colesterol se puede observar que se encuentra dentro de lo normal con un $61,4 \%$; y un $38,6 \%$ se encuentra elevado. La prevalencia del colesterol LDL se encuentra en rangos normales el $10 \%$ de los adultos mayores.

Tabla 3: Distribución perfil lipidico en adultos mayores del geriátrico Miguel de León

\begin{tabular}{llccc}
\hline & & Frecuencia & Porcentaje & $\begin{array}{c}\text { Porcentaje } \\
\text { válido }\end{array}$ \\
\hline \multirow{3}{*}{ Prevalencia de colesterol } & Alto & 17 & 38,6 & 38,6 \\
& Normal & 27 & 61,4 & 61,4 \\
& Total & 44 & 100,0 & 100,0 \\
\hline \multirow{3}{*}{ Prevalencia de trigliceridos } & Alto & 12 & 27,3 & 27,3 \\
& Normal & 32 & 72,7 & 72,7 \\
& Total & 44 & 100,0 & 100,0 \\
\hline \multirow{2}{*}{ Prevalencia de HDL colesterol } & Alto & 17 & 38,6 & 38,6 \\
& Normal & 27 & 61,4 & 61,4 \\
& Total & 44 & 100,0 & 100,0 \\
\hline Prevalencia de LDL colesterol & Normal & 44 & 100,0 & 100,0 \\
\hline
\end{tabular}

Fuente: Base de datos SPSS

En la tabla 4 se puede observar que, en el Geriátrico Miguel de León, existe más prevalencia de diabetes mellitus tipo 2 con un $95,5 \%$ y diabetes mellitus tipo 1 con un $4,5 \%$.

Tabla 4: Distribución de los valores de glucosa en adultos mayores del geriátrico Miguel de León

\begin{tabular}{llcccc}
\hline & & Frecuencia & Porcentaje & $\begin{array}{c}\text { Porcentaje } \\
\text { válido }\end{array}$ & $\begin{array}{c}\text { Porcentaje } \\
\text { acumulado }\end{array}$ \\
\hline \multirow{3}{*}{ Prevalencia de diabetes } & 1 & 2 & 4,5 & 4,5 & 4,5 \\
& 2 & 42 & 95,5 & 95,5 & 100,0 \\
& Total & 44 & 100,0 & 100,0 & \\
\hline
\end{tabular}

Fuente: Base de datos SPSS

Se puede observar que la prevalencia del hemograma en adultos mayores se encuentra en un rango alto con un $15,9 \%$ y bajo un $9,1 \%$, mientras que el hematocrito se encuentra en un rango alto con un $9,1 \%$ y bajo en un $13,6 \%$, se puede observar que los glóbulos blancos se encuentran en un rango bajo con un $34,1 \%$ y alto en un 
9,1\%, los monocitos se encuentran en un $100 \%$ normales. Los neutrófilos se encuentran en rango normal con un $97,7 \%$, los glóbulos rojos se encuentran en un alto en un
$13,6 \%$ y bajo con un $6,8 \%$ las plaquetas y volumen celular medio MCV se encuentran rangos normales (Tabla 5).

Tabla 5: Distribución del hemograma en adultos mayores del geriátrico Miguel de León

\begin{tabular}{|c|c|c|c|c|}
\hline & & Frecuencia & Porcentaje & $\begin{array}{c}\text { Porcentaje } \\
\text { válido }\end{array}$ \\
\hline \multirow{4}{*}{ Prevalencia de hemoglobina } & Alto & 7 & 15,9 & 15,9 \\
\hline & Bajo & 4 & 9,1 & 9,1 \\
\hline & Normal & 33 & 75,0 & 75,0 \\
\hline & Total & 44 & 100,0 & 100,0 \\
\hline \multirow{4}{*}{ Prevalencia de hematocrito } & Alto & 4 & 9,1 & 9,1 \\
\hline & Bajo & 6 & 13,6 & 13,6 \\
\hline & Normal & 34 & 77,3 & 77,3 \\
\hline & Total & 44 & 100,0 & 100,0 \\
\hline \multirow{4}{*}{ Prevalencia de glóbulos blancos } & Alto & 4 & 9,1 & 9,1 \\
\hline & Bajo & 15 & 34,1 & 34,1 \\
\hline & Normal & 25 & 56,8 & 56,8 \\
\hline & Total & 44 & 100,0 & 100,0 \\
\hline \multirow{3}{*}{ Prevalencia de linfocitos } & BAJO & 4 & 9,1 & 9,1 \\
\hline & NORMAL & 40 & 90,9 & 90,9 \\
\hline & Total & 44 & 100,0 & 100,0 \\
\hline Prevalencia de monocitos & Normal & 44 & 100,0 & 100,0 \\
\hline \multirow{3}{*}{ Prevalencia de neutrofilos } & Alto & 1 & 2,3 & 2,3 \\
\hline & Normal & 43 & 97,7 & 97,7 \\
\hline & Total & 44 & 100,0 & 100,0 \\
\hline Prevalencia de MCV & Normal & 44 & 100,0 & 100,0 \\
\hline Prevalencia de plaquetas & Normal & 44 & 100,0 & 100,0 \\
\hline \multirow{4}{*}{ Prevalencia de neutrofilos } & Alto & 6 & 13,6 & 13,6 \\
\hline & Bajo & 3 & 6,8 & 6,8 \\
\hline & Normal & 35 & 79,5 & 79,5 \\
\hline & Total & 44 & 100,0 & 100,0 \\
\hline
\end{tabular}

Fuente: Base de datos SPSS

En la tabla 6 se presenta los resultados de la evaluación global del MNA (Mini Nutritional Assessment), en la cual se encontró que el 9,10\% adultos mayores se encuentran en riesgo de desnutrición, el 22,72 presentó desnutrición y el $68,18 \%$ se encontraron en estado nutricional normal.

Tabla 6: Evaluación del estado nutricional en adultos mayores del geriátrico Miguel de León

\begin{tabular}{lcc}
\hline & Frecuencia & Porcentaje \\
\hline Estado nutricional normal & 30 & $68,18 \%$ \\
Riesgo de desnutrición & 10 & $22,72 \%$ \\
Desnutrición & 4 & $9.10 \%$ \\
Total & 44 & $100 \%$ \\
\hline
\end{tabular}

Fuente: Base de datos SPSS

\section{Discusión}

Los antecedentes clínicos del estudio realizado por $\mathrm{Mu}$ ñoz et al. Muestra que el $74,5 \%$ de las personas padece hipertensión y el $41 \%$ padece artritis degenerativa y diabetes, lo que representa el 34,9\%. Según investigaciones de Macías y Balbuena, encontraron que el 38,3\% tenía hipertensión arterial, el 34,5\% depresión y el $14 \%$ diabetes. A diferencia de nuestro estudio, la prevalencia de hipertensión fue del $30,7 \%$, la prevalencia de diabetes fue del $28,4 \%$ y la prevalencia de ausencia de antecedentes fue del 28,4\%. ${ }^{10}$

En este estudio se encontró que la prevalencia de DM en la población encuestada fue $17.5 \%$. De acuerdo con otras encuestas SABE realizadas en diferentes países de Latinoamérica y del Caribe, la prevalencia de DM se encontró que iba desde el $13 \%$ en Santiago de Chile hasta $22 \%$ en México D.F y Bridgetown. Nuestros resultados son similares a los encontrados en la de la ciudad de Sao Paulo, en donde documentaron una prevalencia de $17 \% .^{11}$

En cuanto a la distribución por edades de la población estudiada, en la encuesta SABE realizada en cada ciudad, Bogotá tiene una mayor similitud con Montevideo y Santiago de Santiago, Chile, debido a que la población menor de 75 años encuesta tiene una mayor proporción de 67,9. \% Bogotá, 66,1\% en Santiago y 69,9\% en Montevideo. En este estudio se demuestra que la correlación entre la edad mayor de 85 años y la DM es pequeña, según el análisis bivariado se encuentra que el rango de pacientes con la enfermedad es mayor, entre 70-74 años. En 2016, igual a la mayor prevalencia reportada por Montevideo y Santiago. ${ }^{11}$ 
Podemos observar en nuestro estudio que existe tres tipos de adultos mayores la prevalencia de edad en adultos mayores es de 75 a 90 con un porcentaje de $47,7 \%$ siendo las viejas o ancianas las que se encuentran en el Geriátrico Miguel de León; seguidas de los adultos mayores de edad avanzada encontrándose entre 60 - 74 años con un 31,8\% y teniendo con el 20,5\% los adultos de más de 90 años, en relación a los rangos de la OPS.

Según la OPS la población de todos los países de las Américas se encuentra en proceso de envejecimiento, aunque en los distintos países envejece a velocidad y en momentos diferentes. La disminución de los indicadores de fertilidad y de mortalidad, fundamentalmente a edades tempranas de la vida, está dando lugar a una transición demográfica muy rápida en la Región. No solo se ha incrementado la proporción de personas mayores en la población del continente, sino que también ha aumentado de manera importante la esperanza de vida al nacer y por edades específicas. Hoy en día, la esperanza de vida a los 60 años se ha calculado en 21 años; el $81 \%$ de las personas que nacen en la Región vivirán hasta los 60 años, mientras que $42 \%$ de ellas sobrepasarán los 80 años de edad. ${ }^{12}$

Los valores de hematocrito, hemoglobina y colesterol sérico, surge un razonamiento similar al utilizado con los valores de albúmina sérica. La hipocolesterolemia $(<160)$ es muy importante como factor de riesgo de mortalidad, en particular asociada a cáncer. En presencia de desnutrición, sugiere que se debe a causas inflamatorias (desgaste), más que a baja ingesta. Así mismo, se puede tomar el recuento total de linfocitos en sangre periférica como indicador de desnutrición, ya que, la primera estructura del sistema inmune, el timo, y estructuras secundarias como el bazo y nódulos linfáticos, se alteran marcadamente en tamaño, peso, arquitectura y la sangre se hace evidente la depleción de linfocitos, disminuyendo su número total. Se mide en forma absoluta o se calcula basado en el recuento total de leucocitos y el porcentaje de linfocitos presentes. En la malnutrición se observan valores por debajo de 1500 linfocitos por mm3. Pero, como en la modificación de este indicador participan otras variables, su interpretación como indicador nutricional es limitado. ${ }^{13}$

En nuestro estudio se observa la prevalencia del hemograma en adultos mayores se encuentra en un rango alto con un $15,9 \%$ y bajo un $9,1 \%$, mientras que el hematocrito se encuentra en un rango alto con un $9,1 \%$ y bajo en un $13,6 \%$, se puede observar que los glóbulos blancos se encuentran en un rango bajo con un $34,1 \%$ y alto en un $9,1 \%$, los monocitos se encuentran en un $100 \%$ normales. Los neutrófilos se encuentran en rango normal con un $97,7 \%$, los glóbulos rojos se encuentran en un alto en un $13,6 \%$ y bajo con un $6,8 \%$ las plaquetas y volumen celular medio MCV se encuentran rangos normales.

En relación al estado nutricional en el estudio realizado por Josefa Danielma Lopes, detectado por MEN®, el presente estudio demostró que una parte considerablemente alta de los ancianos estaban en riesgo de desnutrición o desnutridos, dato este corroborado con otro estudio que utiliza la MEN® como una de las maneras para evaluar el estado nutricional de los ancianos, que mostró que las mujeres presentaron $31.8 \%$ de desnutrición y $50.0 \%$ riesgo de desnutrición similares a los hombres, que presentaron $27.0 \%$ y $40.0 \%$, respectivamente. Sin embargo, en el presente estudio la diferenciación no se hizo por sexo, sino por el número de ancianos. En una revisión sistemática de la literatura sobre indicadores antropométricos del estado nutricional en el anciano se constató que el MEN® se utilizó en nueve estudios y demostró un valor pronóstico de la desnutrición de $97,0 \%{ }^{14}$

En nuestro estudio se presenta los resultados de la evaluación global del MNA (Mini Nutritional Assessment), en la cual se encontró que el $9,10 \%{ }^{4}$ adultos mayores se encuentran en riesgo de desnutrición, el 22,72\% ${ }^{10}$ presentó desnutrición y el $68,18 \%$ se encontraron en estado nutricional normal.

\section{Conclusión}

Se puede observar que en la ciudad de Cuenca existe más adultos mayores institucionalizados en un rango de 70 a 95 años son viejas o ancianas y se encuentra en relación al rango de la Organización Panamericana de Salud. Con los cambios fisiológicos que se va desarrollando en el ser humano se puede observar que el individuo al llegar a ser adultos mayores existe un cambio en los perfiles lipídicos donde se puede observar que se relacionan con otros estudios, donde se debería es tener una mejor nutrición para poder evitar problemas de salud. Con el paso de los años el examen de sangre se puede verificar varios cambios del hematocrito ya que el mismo disminuye y puede provocar problemas de salud como la anemia q es muy prevalente y se tiene que prevenir con una mejor alimentación y mejor calidad de vida del adulto mayor.

\section{Fuente de Financiamiento}

Este estudio es autofinanciado.

\section{Conflicto de Intereses}

No existen conflictos personales, profesionales, financieroso de otro tipo.

\section{Consentimiento Informado}

Los autores cuentan con el consentimiento informado de los pacientes para la investigación, la publicación del caso y sus imágenes.

\section{Referencias Bibliográficas}

1. Alvarado García AM, Salazar Maya ÁM. Aging concept analysis [Internet]. Vol. 25, Gerokomos. Spanish Publishers Associate; 2014 [citado el 4 de febrero de 2021]. p. 57-62. Disponible en: http://scielo.isciii.es/scielo.php?script=sci_arttext\&pid= S1134-928X2014000200002\&lng=es\&nrm=iso\&tlng=es 
2. Martínez Pérez T, González Aragón C, Castellón León Recibido: 11 de octubre de 2020 G, González Aguiar B. El envejecimiento, la vejez y la calidad de vida: ¿éxito o dificultad? Rev Finlay [Internet]. 2018 [citado el 4 de febrero de 2021];8(1):59-65. Disponible en: http://scielo.sld.cu/scielo.php?script=sci_arttext\& pid=S2221-24342018000100007

3. M RR. Valoración de factores biopsicosociales determinantes del riesgo de desnutrición en los adultos mayores en hogares de ancianos (Tesis). An la Fac Ciencias Médicas. 2015;48(2):17-32.

4. Tafur Castillo J, Guerra Ramírez M, Carbonell A, Ghisays López M. Factores que afectan el estado nutricional del adulto mayor. Latinoam Hipertens [Internet]. 2018;13(5). Disponible en: http://www.revhipertension. com/rlh_5_2018/factores_que_afectan_el_estado.pdf

5. Cuerda C, Álvarez J, Ramos P, Abánades JC, GarcíaDe-lorenzo A, Gil P, et al. Prevalence of malnutrition in subjects over 65 years of age in the community of Madrid. The DREAM + 65 study. Nutr Hosp. 2016;33(2):263-9.

6. Cordero R, Fontanillo P. Health status of the elderly. Current situation.

7. Musso AM. Anemia en el adulto mayor. Acta Bioquim Clin Latinoam [Internet]. 2017;51(3):319-24. Disponible en: https://www.redalyc.org/pdf/535/53553013006.pdf

8. Alvarado-García A, Lamprea-Reyes L, Murcia-Tabares K. La nutrición en el adulto mayor: una oportunidad para el cuidado de enfermería. Enfermería Univ. 2017;14(3):199-206.

9. Barrios JC, Torres AC, Vélez YF, Castellanos LB, Labrada PG, Hernández EM. Alimentación no saludablesedentarismo-estrés asociados con HTA e IAM: Reporte de casos. FACSALUD-UNEMI [Internet]. el 15 de diciembre de 2020 [citado el 4 de febrero de 2021];4(7):3-10. Disponible en: http://ojs.unemi.edu.ec/ index.php/facsalud-unemi/article/view/1160

10. Nestle Nutrition Institude. What is the MNA®? 2020;1-3.

11. Morros-González E, Borda MG, Reyes-Ortiz C, Chavarro-Carvajal D, Cano-Gutierrez C. Anciano con Diabetes y factores asociados. Acta Médica Colomb. 2017;42(4):230-6.

12. Loredo-Silva MT. Estado de salud de la población universitaria. Salud Publica Mex. 2015;19(6):867-92.

13. Faisant C, Lauque S GY. Valoración Nutricional Del Adulto Mayor. Parte I Módulos Valoración Clínica. 2009;12.

14. Ferreira Lopes JD, Guimarães Oliveira Soares MJ, Lidiane Jácome de Lima C, Maynnard Costa Ferreira T, Simplício de Oliveira P, Alves da Silva M. Evaluación nutricional por el Mini Nutritional Assessment: una herramienta para las enfermeras TT - Avaliação nutricional pela Mini avaliação Nutricional: uma ferramenta para o enfermeiro TT - Nutritional assessment by the Mini-Nutritional Assessment: a. Enferm glob. 2018;17(51):267-305. 